\title{
ENCP: a new Energy-efficient Nonlinear Coverage Control Protocol in mobile sensor networks
}

\author{
Zeyu Sun ${ }^{1,2^{*}}$, Guozeng Zhao ${ }^{1}$ and Xiaofei Xing ${ }^{3}$
}

\begin{abstract}
The node deployment in mobile sensor networks (MSNs) is mostly performed in a random method. However, a large number of redundant nodes may exist due to the randomness. As a result, severe data congestion may be caused and the quality of coverage $(\mathrm{Q} O \mathrm{C})$ is undermined. In order to solve this $\mathrm{QoC}$ problem, we propose an Energy-efficient Nonlinear Coverage Control Protocol (ENCP). This protocol utilizes the normal distribution to calculate the minimal number of sensors which is required to guarantee coverage over the monitoring area. We also balance the node energy consumption and achieve the collaborative scheduling among all the sensor nodes. Meanwhile, when a certain QoC is guaranteed, we present the calculation model for the normal distribution of the sensing ranges and the proportional relationship between different parameters in the QoC function. Finally, simulation results show that the ENCP could not only improve the network QoC and network coverage rate but also effectively control the energy exhaustion at the nodes. Therefore, the network lifetime can be effectively prolonged.
\end{abstract}

Keywords: Mobile sensor network, Nonlinear coverage, Quality of coverage, Networks coverage rate, Network lifetime

\section{Introduction}

Mobile sensor networks (MSNs) are composed of tens of thousand sensor nodes whose processing ability, communication bandwidth, and energy are quite limited. Usually, the high-density node deployment strategy is employed to improve the network performance [1]. The node density can be as high as 200 nodes $/ \mathrm{m}^{2}$. However, a series of problems are brought about in terms of scalability, information redundancy, wireless channel interference, and energy wastes. Since the sensors are mostly battery-powered, it is often impractical to replace the batteries due to the limits of the application environment. However, the network is expected to work over a long period of time without further energy supply, which makes the energy management a key problem for the study of sensor networks.

\footnotetext{
*Correspondence: lylgszy@163.com

${ }^{1}$ School of Computer Science and Engineering, Luoyang Institute of Science and Technology, Luoyang, China

${ }^{2}$ Department of Computer Science and Technology, Xi'an Jiaotong

University, Xi'an, China

Full list of author information is available at the end of the article
}

The node scheduling enables the switching between different states of the nodes, utilizing the node redundancy and the heuristic algorithms [2,3]. The aim of the node scheduling is to save the node energy and prolong the network lifetime with the desired QoC by shutting down the nodes in turn. Currently, most coverage control algorithms obtain the coverage information according to the location information of the nodes [4-6]. However, the acquisition of location information relies on external infrastructures such as the GPS and directional antennas, which greatly increases the hardware cost and energy consumption for the nodes. Meanwhile, locating inaccuracy cannot be avoided. It is hard for the location-based coverage control algorithms to obtain the accurate coverage relationship between the nodes without precise location information [7-9]. Furthermore, shutting down parts of the nodes may lead to coverage dead zones and inaccuracy for the network monitoring. Hence, there is a growing interest on the study of coverage control algorithms with no location information required [10-12]. But these algorithms cannot achieve full coverage. Fortunately, the full coverage over the monitoring area is not necessarily needed for the sensor network for 
most applications $[13,14]$. It is sufficient for the network to maintain a reasonable coverage rate. In other words, only partial coverage is required and the coverage rate is one of the indicators to evaluate the network QoC. Partial coverage control strategies could not only simplify the network protocol design but also improve the flexibility of the network configuration. The users could achieve a different tradeoff between the energy consumption and the QoC according to their own needs. After the deployment of the same kind of sensor nodes in the realistic environment, the actual sensing range would be higher or lower than the rated sensing range due to the influence of its own characteristics or the environment. As a result, the sensing range for the sensor node becomes different [15]. The massive numbers of sensor nodes deployed in the sensing field accomplish the data acquisition in a self-organized way. After certain processing, the acquired data is transmitted to the base station via multi-hops among the nodes. This process is depicted in Fig. 1.

The sensing characteristics for the same kind of sensors are investigated in the realistic environment. We also study the coverage problem for the randomly deployed sensor network where the sensing ranges for the nodes satisfy the normal distribution. The calculation model with no location information required is presented for the redundancy degree. Also elaborated is the calculation model for the minimal number of working nodes with a desired QoC. Based on QoC problem, we present a new Energy-efficient Nonlinear Coverage Control Protocol (ENCP) in mobile sensor networks. Under the premise of a certain QoC, we can use the minimum sensor node to complete the effective coverage of the monitoring area, thus suppress to the network energy consumption and a longer network lifetime.

\section{Related work}

Dong et al., node scheduling scheme using spatial resolution of wireless sensor networks [16]. The sensors calculate the sponsored area by the neighbors according to the location information. Then, the coverage relationship can be derived. However, this algorithm fails to consider the case of overlapped sensing area, which may cause a large number of working nodes and extra energy waste. Xing et al. proposed target coverage scheme using linear programming [17]. The probing method is as follows. Every sleeping node would regularly check whether working nodes exist within its probing area. If no, this sleeping node will switch to the work state. Otherwise, it will remain sleeping. Obviously, some nodes may keep working consistently according to the PEAS protocol, which leads to an early node failure and unbalanced network energy consumption. Therefore, the QoC is affected. Wang et al. proposed saluting of scheduling mobile sensor and fixed sensors for target tracking algorithm. (MTTA) [18]. The node closest to the optimal location is chosen as the working node according to this protocol. Therefore, the QoC is guaranteed with the smallest number of working nodes. However, the calculation of the optimal location relies on rigorously accurate positioning technique and high computational complexity. Sun et al. proposed a linear

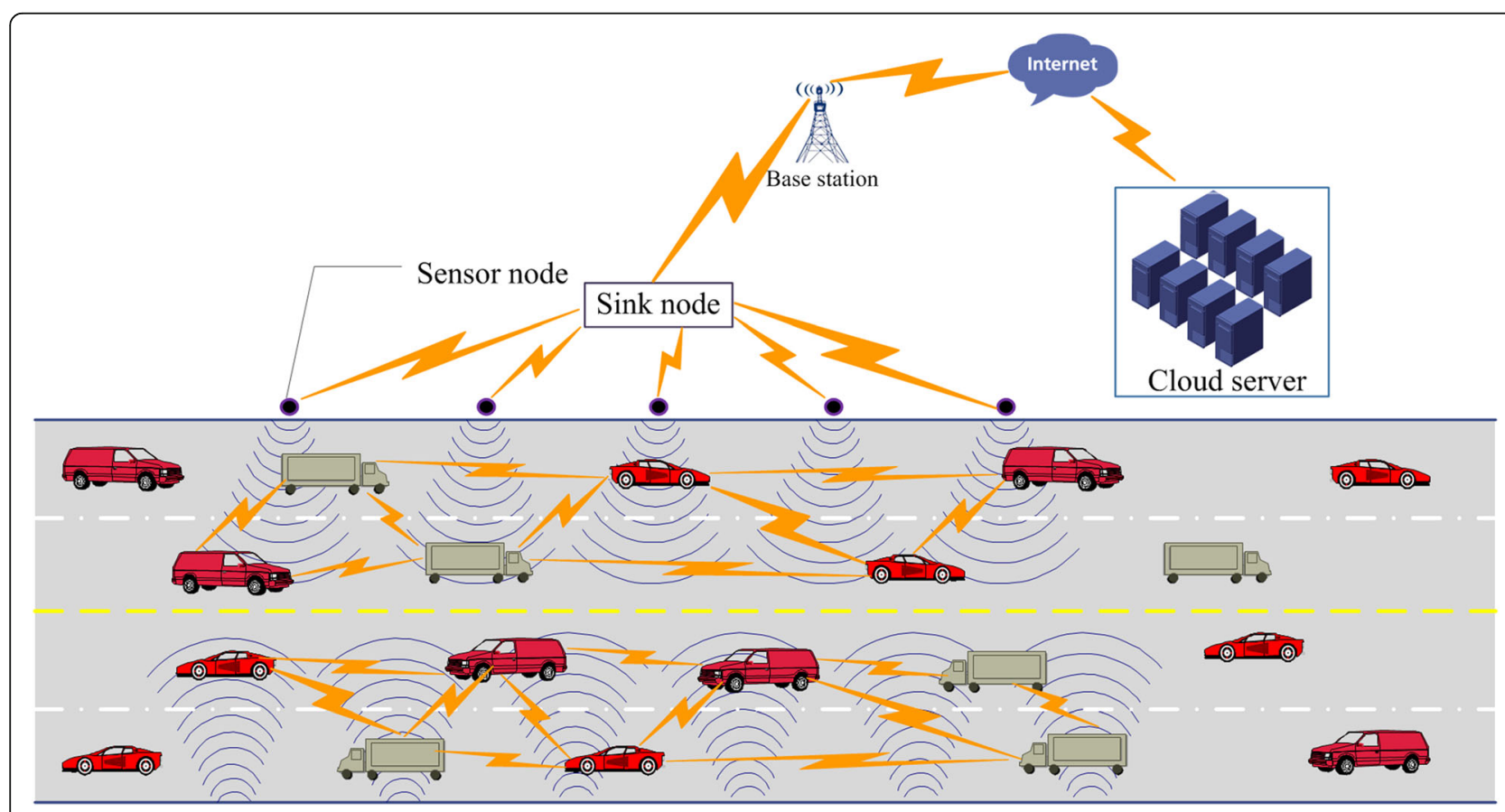

Fig. 1 Coverage control structure of mobile sensor networks 
programming optimization coverage scheme (LPOCS) [19] which divides all of the sensor nodes into many subsets. The connectivity and coverage for a specific target within every subset can then be guaranteed.

Energy-efficient sleep schedule with service coverage guarantee is firstly given by Zhang et al. in [20]. The mathematical relationships among the QoC, the number of working nodes, the size of the sensing area, and the sensing ranges of the sensor nodes are derived while no location information is required in this algorithm. Based on the mathematical relationship, three scheduling strategies are proposed, i.e., Efficient Coverage Algorithm Based on Node Scheduling Strategy (ECNSS) [21], Energy-efficient Multi-target Coverage Control Protocol (EMCCP) [22], and Efficient Rendezvous algorithms for mobility-enabled (ERME) [23]. In these three scheduling schemes, the working nodes are chosen in completely a random method, which may result in the instability of the QoC. Furthermore, the limited delay mechanism is employed in the scheduling schemes to ensure that all the data can be collected within the monitoring area. Liu et al. [24] derived the mathematical relationships among the QoC, the size of the network monitoring area, the sensing range of the nodes, and the node density. Again, the location information is not needed for the QoC during the derivation. Also included are three methods to evaluate the coverage, i.e., partial coverage, node coverage, and monitoring ability. A further modification was made based on the protocol in [25], and the energy balance strategy was introduced. The working nodes are chosen according to the remained energy, and optimization is performed on the distribution of working nodes. Therefore, the network lifetime is further prolonged. An extension of the results in [26] was made in [27], and the influence of edge effects is considered. As a result, the analytical results are more accurate and many multiple-fold coverage problems can be analyzed. Zeng et al. [28] studied the coverage problem for the sensor network where the node deployment satisfies the twodimension normal distribution. Wu et al. [29] proposed a probability-based calculation method for the node redundancy degree, according to which, the node could independently calculate its own redundancy degree. Furthermore, a node scheduling protocol, lightweight deployment-aware scheduling (LDAS) is proposed with no location information required. However, the sponsorship of the two-hop neighbors for the sensing area of the nodes is neglected. As a result, many of the working nodes chosen by the LDAS algorithm are redundant. A joint scheduling algorithm is proposed by Chen et al. [30] which adds more working nodes to guarantee the network connectivity with the required QoC.

Cheng et al. derived the minimal number of working nodes and the corresponding node deployment location for the simultaneous guarantee of $k$ coverage and network connectivity [31]. The QoC and connectivity quality are guaranteed with the minimal number of working nodes by deploying the mobile sensor nodes to the designated positions. Therefore, the network energy is saved. Lu et al. presented the sensor node deployment scheme which guarantees the full coverage over the monitoring area and the network 2 connection [32]. It was also proven that the proposed deployment is optimal regardless of the value of $R_{c} / R_{s}$ ( $R_{c}$ is the communication range and $R_{s}$ is the sensing range).

All the studies stated above are based on different assumptions [33-37]. The sensing range of the node varies according to its own characteristics and the influence of other external factors such as the environment. This paper focuses on the realistic sensing characteristics of randomly deployed MSNs and studies the coverage problem for the MSNs where the sensing range of the node satisfies the normal distribution and no location information is required.

\section{Network model and problem description}

\subsection{Basic assumptions}

Assuming: All the sensor nodes are randomly deployed within a two-dimension area $M$. Furthermore, these MSNs exhibit the following characteristics

(1)The node density is sufficiently high. When all the nodes are working nodes, the QoC can be guaranteed and a large number of redundant nodes will exist in the mobile sensor network.

(2)Boolean sensing model is adopted by the nodes. In other words, if the sensing range of an arbitrary node $i$ is $R_{i}$, then, the sensor area of node $i$ is circle with center $i$ and radius $R_{i}$. The sensing area is denoted as $\Theta i\left(R_{i}\right)$.

(3) The sensing ranges for all the nodes in the network satisfy the normal distribution $N\left(R_{0}, \delta^{2}\right)$ where $R_{0}$ is the average of the sensing ranges, i.e., the rated sensing range, $\delta$ is the standard deviation, and $R_{0} \geq 3.3 \delta$ so that the sensing ranges concentrate in the interval $\left[0,2 R_{0}\right]$.

(4) The monitoring area is large enough so that the boundary effect can be neglected.

(5) The communication range of the node is no smaller than $4 R_{0}$, i.e., the communication range is at least twice times the maximal sensor range.

(6) No GPS or other locating techniques are required for each node to acquire the location information.

(7) The sensor nodes are randomly deployed in the monitoring areas with high density, and the sensor nodes are independent from each other. 
In large scale MSNs, the random deployment scheme is both practical and low in cost [38-40]. Therefore, the random deployment scheme is employed in this network model. Although all the nodes have been the same rated sensor range $R_{0}$ in the binary sensing model, the actual sensing range would still be affected by the characteristics of the nodes and the environment. Therefore, we employ the normal assumption $N\left(R_{0}, \delta^{2}\right)$ on the distribution of the actual sensing range. Since the actual sensing range is no smaller than 0 , we have the limitation $R_{0} \geq 3.3 \delta$ so that the probability that the actual sensing range lies in $[-\infty, 0]$ is smaller than $5 \times 10^{-5}$. Due to the symmetry of the normal distribution, we can derive that the probability that the actual sensing range lies in $[0$, $\left.4 R_{0}\right]$ is larger than $99.99 \%$, which approximately makes it a certain event.

\subsection{Definitions and problem description}

Definition 1 An arbitrary node $i$, its neighbor sensor set is defined as:

$$
S(i, j)=\left\{(i, j) \in S \mid d(i, j) \leq R_{i}+R_{j}, j \in S, i \neq j\right\}
$$

where $S$ is the set of all the nodes deployed within the monitoring area $M, d(i, j)$ is the Euclidean distance between sensor node $i$ and sensor node $j, R_{i}$, and $R_{j}$ are the sensing ranges of sensor node $i$ and sensor node $j$, respectively.

Definition 2 For an arbitrary sensor node $i$, the sensor node redundancy degree has been defined as the ratio of overlapped monitoring area to its own monitoring area, i.e.,

$$
\xi(i)=\operatorname{area}\left(\left(\underset{j \in(S(i) \cap \phi)}{\cup} S_{j}\right) \cap S_{i}\right) / \operatorname{area}\left(S_{i}\right)
$$

where $\phi$ is the set of all the working sensor nodes, $S_{i}$ and $S_{j}$ are the monitoring area for sensor node $i$ and sensor node $j$, respectively, area $\left(\left(\underset{j \in(S(i) \cap \phi)}{\cup} S_{j}\right) \cap S_{i}\right)$ is the overlapped monitoring area, and $S_{i}$ is the monitoring area for node $i$.

Definition 3 QoC is defined as the ratio of the combination of the monitoring area, which all the working nodes are monitoring area $M$.

$$
\eta=\operatorname{area}\left(\left(\cup_{i \in \phi} S_{i}\right) \cap M\right) / \operatorname{area}(M)
$$

where $\phi$ is the set of all the working nodes, $S_{i}$ is the monitoring area for node $i$, area $(M)$ is the size of the whole monitoring area, area $\left(\left(\cup_{i \in \phi} S_{i}\right) \cap M\right)$ is the intersection between $M$ and the monitoring area of all the working nodes. As a matter of fact, the quality of coverage is also equivalent to the network coverage probability.

Definition 4 The effective network lifetime has been defined as the total time which the required coverage quality can be guaranteed.

$$
T=\sum_{j \in\{j \mid j \in S \cap \eta\}} \Delta t_{i}
$$

where $\Delta t_{i}$ is a time slice which is close to $0, \Delta t_{i+1}$ is the subsequent slice after $\Delta t_{i}, \eta_{o}^{\Delta t_{j}}$ is the quality of coverage obtained within $\Delta t_{j}$, and $\eta_{d}$ is the desired QoC.

It is assuming that a large number of working sensor nodes are randomly deployed within the sensing area $M$, and the sensing ranges of all the sensor nodes must satisfy the normal distribution $N\left(R_{0}, \delta^{2}\right)$. Furthermore, $R_{0} \geq 3.3 \delta$. All the redundant nodes are shut down on the condition that the desired $\mathrm{QoC} \eta_{d}$ is satisfied, i.e., a minimal set of working nodes $\phi$ is found which can guarantee the satisfaction of $\operatorname{area}\left(\left(\cup_{i \in \phi} S_{i}\right) \cap M\right) / \operatorname{area}(M) \geq \eta_{d}$. A calculation model is constructed for the node redundancy degree. We also design the node scheduling strategy for the set $\phi$, which can be employed to prolong the effective network lifetime.

\section{Problem analysis and research methods}

\subsection{ENCP problem solution}

Two questions have to be answered for the node scheduling strategy in the MSNs. The first one is how to determine whether a node is redundant [41-43]. The second one is the specific scheduling of the nodes. We mainly deal with the first question here while the second one is left for the next section. The calculation of node redundancy degree based on location information employs the geometry knowledge and offers the accurate coverage relationship between nodes [44-46]. However, when the location information is not available, it is hard for the nodes to derive the node redundancy degree. Still, we could still utilize the number of neighbor sensors of a node and calculate the expectation for the node redundancy degree based on the probability theory.

Theorem 1 The coverage probability that one sensor node within the sensing range is covered by one of the neighbor sensors nodes are:

$$
P=\frac{R_{0}^{2}}{4\left(R_{0}^{2}-\delta^{2}\right)}
$$

Proof Assuming that the sensing range of node $a$ is $R_{a}$, and the sensing area is $\Theta_{1} a\left(R_{a}\right)$ while the sensing range of node $b$ is $R_{b}$ and the sensing area is $\Theta b\left(R_{b}\right)$. If node $b$ is a working neighbor sensor of node $a, d(a, b) \leq R_{a}+$ $R_{b}$, i.e., node $b$ must lie within the circle $\Theta_{2} a\left(R_{a}+R_{b}\right)$. 
This circle has center $a$ and radius $R_{a}+R_{b}$. If an arbitrary node $q$ within $\Theta_{1} a\left(R_{a}\right)$ is covered by the sensing area $\Theta b\left(R_{b}\right)$, node $b$ must lie within the circle $\Theta q\left(R_{b}\right)$ which has center $q$ and radius $R_{b}$. The relationships among $\Theta_{1} a\left(R_{a}\right), \Theta_{2} a\left(R_{a}+R_{b}\right)$, and $\Theta q\left(R_{b}\right)$ are depicted in Fig. 2.

Since all the sensor nodes are randomly deployed within the monitoring area $M$, the probability is 1 /area $(M)$ that each node is deployed at a certain point in $M$. Therefore, the probability of $P_{b-a}$ that one node within the sensing area of sensor node $a$ is covered by a neighbor sensor node $b$ is

$$
\begin{aligned}
P_{b-a} & =\frac{\operatorname{area}\left(R q\left(R_{b}\right)\right) \cdot(1 / \operatorname{area}(M))}{\operatorname{area}\left(R a_{2}\left(R_{a}+R_{b}\right)\right) \cdot(1 / \operatorname{area}(M))} \\
& =\frac{R_{b}^{2}}{\left(R_{a}+R_{b}\right)^{2}}
\end{aligned}
$$

Since the sensing ranges of all the sensor nodes must satisfy the normal distribution $N\left(R_{0}, \delta^{2}\right)$ with $R_{0} \geq 3.3 \delta$, the two-dimension random variable $\left(R_{a}, R_{b}\right)$ satisfies the two-dimension normal distribution $N\left(R_{0}, \delta^{2} ; R_{0}, \delta^{2}, 0\right)$. Therefore, the expectation of $P_{b-a}$ can be derived as:

$$
E\left(P_{b-a}\right)=\frac{1}{2 \pi \delta^{2}} \int_{0}^{2 R_{0}} \int_{0}^{2 R_{0}} \frac{R_{b}^{2}}{\left(R_{a}+R_{b}\right)^{2}} \cdot \mathrm{e}^{-\frac{\left(R_{a}-R_{0}\right)^{2}+\left(R_{b}-R_{0}\right)^{2}}{2 \delta^{2}}} \mathrm{~d} R_{a} \mathrm{~d} R_{b}
$$

Set $x_{1}=R_{a}-R_{b}, x_{2}=R_{b}-R_{a}$, we have

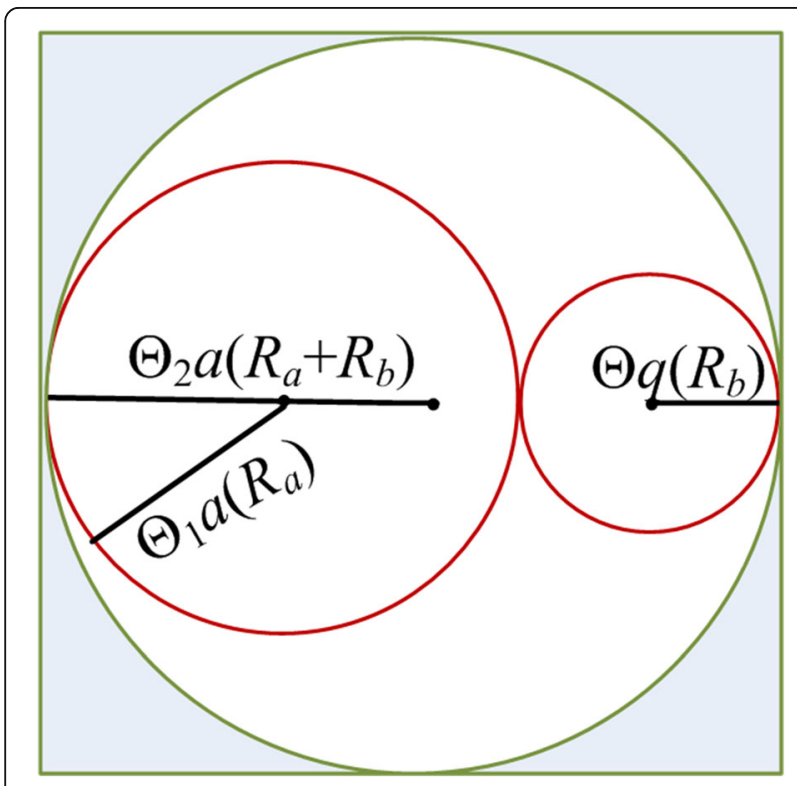

Fig. 2 Illustration of the locations of the circles

$$
\begin{aligned}
E\left(P_{b-a}\right)=\frac{1}{2 \pi \delta^{2}} & \left\{\underset{R o\left(R_{0}\right)}{ }\left(\frac{R_{0}+x_{2}}{2 R_{0}+x_{1}+x_{2}}\right)^{2} \cdot \mathrm{e}^{-\frac{x_{1}^{2}+x_{2}^{2}}{2 \delta^{2}}} \mathrm{~d} x_{1} \mathrm{~d} x_{2}\right. \\
& \left.+\iint_{S}\left(\frac{R_{0}+x_{2}}{2 R_{0}+x_{1}+x_{2}}\right) \cdot e^{-\frac{x_{1}^{2}+x_{2}^{2}}{2 \delta^{2}}} \mathrm{~d} x_{1} \mathrm{~d} x_{2}\right\}
\end{aligned}
$$

where the integral regions $N$ and $R o\left(R_{0}\right)$ is depicted in Fig. 3.

According to the derivation below,

$$
\begin{aligned}
\frac{1}{2 \pi \delta^{2}} \iint & \left.\int\left(\frac{R_{0}+x_{2}}{2 R_{0}+x_{1}+x_{2}}\right)^{2} \cdot \mathrm{e}^{-\frac{x_{1}^{2}+x_{2}^{2}}{2 \delta^{2}}} \cdot R_{0} \frac{1}{2 \pi \delta^{2}} \cdot \mathrm{e}^{-\frac{x_{1}^{2}+x_{2}^{2}}{2 \delta^{2}}}\right\} \mathrm{d} x_{1} \mathrm{~d} x_{2} \\
& =\frac{1}{2 \pi \delta^{2}} \int_{-R_{0}}^{R_{0}} \int_{-R_{0}}^{R_{0}} \mathrm{e}^{-\frac{x_{1}^{2}+x_{2}^{2}}{2 \delta^{2}}} \mathrm{~d} x_{1} \mathrm{~d} x_{2}-\frac{1}{2 \pi \delta^{2}} \int_{0}^{(5)_{0}} \int_{0}^{2 \pi} r \cdot e^{-\frac{r^{2}}{2 \delta^{2}}} \mathrm{~d} \theta \mathrm{d} r \\
& =\left(F(x) \mid \begin{array}{l}
R_{0} / \delta \\
-R_{0} / \delta
\end{array}\right)^{2}-\mathrm{e}^{x} \mid \begin{array}{l}
0 \\
-R_{0} /\left(2 \delta^{2}\right)
\end{array}
\end{aligned}
$$

where $F(x)$ is the standard normal distribution function. Set $a(x)=\left(F(x)-F(-x)^{2}-\left(1-\mathrm{e}^{-x 2 / 2}\right)\right)$. Since

$$
\frac{d_{a(x)}}{d_{x}}=\frac{4}{\sqrt{2 \pi}}(F(x)-F(-x)) \cdot \mathrm{e}^{-x^{2} / 2}-x \mathrm{e}^{-x^{2} / 2}<\left(\frac{4}{\sqrt{2 \pi}}-x\right) \cdot \mathrm{e}^{-x^{2} / 2}<0
$$

$a(x)$ is monotonically decreasing in $[3.3,+\infty)$. Since $R_{0} / \delta \geq 3.3$,

$$
\left(F(x) \mid \begin{array}{l}
R_{0} / \delta \\
-R_{0} / \delta
\end{array}\right)^{2}-\mathrm{e}^{x} \mid \begin{aligned}
& 0 \\
& -R_{0} /\left(2 \delta^{2}\right)
\end{aligned}=a\left(R_{0} / \delta\right) \leq a(3.3)=0.0023,
$$

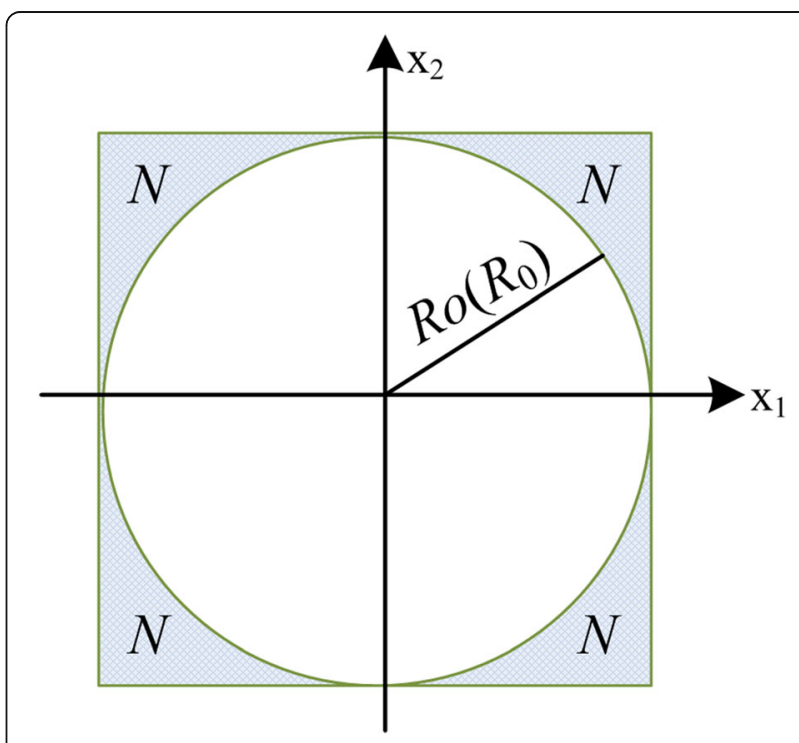

Fig. 3 Illustration of integral regions 
i.e., $\quad \frac{1}{2 \pi \delta^{2}} \iint_{N}\left(\frac{R_{0}+x_{2}}{2 R_{0}+x_{1}+x_{2}}\right)^{2} \cdot \mathrm{e}^{-\frac{x_{1}^{2}+x_{2}^{2}}{2 \delta^{2}}} \mathrm{~d} x_{1} \mathrm{~d} x_{2} \quad$ is small enough to be neglected. Therefore, we have

$$
\begin{aligned}
E\left(P_{b-a}\right) & =\frac{1}{2 \pi \delta^{2}} \iiint_{R o\left(R_{0}\right)}\left(\frac{R_{0}+x_{2}}{2 R_{0}+x_{1}+x_{2}}\right)^{2} \cdot \mathrm{e}^{-\frac{x_{1}^{2}+x_{2}^{2}}{2 \delta^{2}}} \mathrm{~d} x_{1} \mathrm{~d} x_{2} \\
& =\frac{1}{2 \pi \delta^{2}} \int_{0}^{R_{0}} r \cdot \mathrm{e}^{-\frac{r^{2}}{2 \delta^{2}}} \int_{0}^{2 \pi}\left(\frac{R_{0}+\cos \theta}{2 R_{0}+r \sin \theta+r \cos \theta}\right)^{2} \mathrm{~d} \theta \mathrm{d} r
\end{aligned}
$$

Set $f(r)=\int_{0}^{2 \pi}\left(\frac{R_{0}+r \cos \theta}{2 R_{0}+r \sin \theta+r \cos \theta}\right)^{2} \mathrm{~d} \theta$ and the independent variable $\phi=(\pi / 2)-\theta$, so

$$
\begin{aligned}
f(r) & =\int_{-3 \pi / 2}^{\pi / 2}\left(\frac{R_{0}+r \sin \phi}{2 R_{0}+r \sin \phi+r \cos \phi}\right)^{2} \mathrm{~d} \phi \\
& =\int_{0}^{2 \pi}\left(\frac{R_{0}+r \sin \theta}{2 R_{0}+r \sin \theta+r \cos \theta}\right)^{2} \mathrm{~d} \theta
\end{aligned}
$$

Again, we set

$$
g(r)=\int_{0}^{2 \pi} \frac{\left(R_{0}+r \cos \theta\right) \cdot\left(R_{0}+r \sin \theta\right)}{\left(2 R_{0}+r \sin \theta+r \cos \theta\right)^{2}} \mathrm{~d} \theta
$$

So, it can be derived that

$$
\left\{\begin{array}{l}
2 f(r)+2 g(r)=\int_{0}^{2 \pi} \mathrm{d} \theta=2 \pi \\
2 f(r)-2 g(r)=\int_{0}^{2 \pi} \frac{r^{2}(1-2 \sin \theta \cos \theta)}{\left(2 R_{0}+r \sin \theta+r \cos \theta\right)^{2}} \mathrm{~d} \theta
\end{array}\right.
$$

To solve the equation set (8), the right hand side integral of the second equation has to be derived.

$$
\begin{aligned}
\int_{0}^{2 \pi} & \frac{r^{2}(1-2 \sin \theta \cos \theta)}{\left(2 R_{0}+r \sin \theta+r \cos \theta\right)^{2}} \mathrm{~d} \theta=\int_{0}^{2 \pi}(r \sin \theta-r \cos \theta) \mathrm{d}\left(2 R_{0}+r \sin \theta+r \cos \theta\right)^{-1} \\
& =\left.\frac{r \sin \theta-r \cos \theta}{2 R_{0}+r \sin \theta+r \cos \theta}\right|_{0} ^{2 \pi}+\int_{0}^{2 \pi} \frac{r \sin \theta+r \cos \theta}{2 R_{0}+r \sin \theta+r \cos \theta} \mathrm{d} \theta \\
& =\int_{0}^{2 \pi} \frac{2 R_{0}}{2 R_{0}+\sqrt{2} \sin (\pi / 4+\theta)} \mathrm{d} \theta-2 \pi=c \int_{0}^{2 \pi} \frac{1}{c+\sin \phi} \mathrm{d} \phi-2 \pi
\end{aligned}
$$

where $c=\sqrt{2} R_{0} / r$. We further set $t=\tan (\phi / 2)$, so $\phi=$ $2 \arctan t, \mathrm{~d} \phi=2 /\left(1+t^{2}\right) \mathrm{d} t$. Therefore,

$$
\begin{aligned}
2 f(r)-2 g(r) & =c \int_{-\infty}^{+\infty} \frac{2}{c t^{2}+2 t+c} \mathrm{~d} t-2 \pi \\
& =\left.\frac{2 c^{2}}{c^{2}-1} \arctan \left(\frac{c t+1}{\sqrt{c^{2}-1}}\right)\right|_{-\infty} ^{+\infty}-2 \pi \\
& =\frac{4 R_{0}^{2} \pi}{2 R_{0}^{2}-r^{2}}-2 \pi
\end{aligned}
$$

Solving the equation set (13), we have $f(r)=R_{0}^{2} \pi /$ $\left(2 R_{0}^{2}-r^{2}\right)$. Substitute $f(r)$ into (8),

$$
E\left(P_{b-a}\right)=\frac{1}{2 \pi \delta^{2}} \int_{0}^{R_{0}} r \cdot e^{-\frac{r^{2}}{2 \delta^{2}}} \cdot \frac{\pi R_{0}^{2}}{2 R_{0}^{2}-r^{2}} \mathrm{~d} r
$$

In order to further simplify Eq. (14), set $t=-r^{2} / 2 \delta^{2}$, $c_{1}=R_{0}^{2} / \delta^{2}$. So, $E\left(P_{b-a}\right)=\frac{c_{1}}{4} \int_{-\frac{c_{1}}{2}}^{0} \frac{\mathrm{e}^{t}}{t+c_{1}} \mathrm{~d} t=\frac{c_{1}}{4 \mathrm{e}^{c_{1}}} \int_{\frac{c_{1}}{2}}^{c_{1}} \frac{\mathrm{e}^{t}}{t} \mathrm{~d} t$.

According to the McLaurin expansion of $e^{t}$, i.e., $\mathrm{e}^{t}$ $=\sum_{n=0^{\infty}} \frac{t^{n}}{n !}$, we have

$$
\begin{aligned}
E\left(P_{b-a}\right) & =\frac{c_{1}}{4 \mathrm{e}^{c_{1}}} \int_{\frac{c_{1}}{2}}^{c_{1}} \sum_{n=0}^{\infty} \frac{t^{n}}{t n !} \mathrm{d} t \\
& =\frac{c_{1}}{4 \mathrm{e}^{c_{1}}}\left(\ln t \mid \begin{array}{l}
c_{1} \\
\left.\frac{c_{1}}{2}+\left(\sum_{n=1}^{\infty} \frac{t^{n}}{n n !}\right) \mid \begin{array}{l}
c_{1} \\
\frac{c_{1}}{2}
\end{array}\right)
\end{array}\right.
\end{aligned}
$$

Set $I_{1}=\sum_{n=1}^{\infty} \frac{t^{n}}{n n !}$, we have $t I_{1}-I_{1}=-t+\sum_{n=2}^{\infty} \frac{t^{n}}{n !}$ $+\sum_{n=2}^{\infty} \frac{t^{n}}{(n-1) n n !}$. So, $I_{1}=\frac{\mathrm{e}^{t}-3}{t-1}-2+\sum_{n=2}^{\infty} \frac{t^{n}(t-1)^{-1}}{(n-1) n n !}$

Substituting $I_{1}$ into Eq. (15), we have

$$
\begin{aligned}
E\left(P_{b-a}\right) & =\frac{c_{1}}{4 \mathrm{e}^{c_{1}}}\left\{\ln 2+\frac{\mathrm{e}^{c_{1}}}{c_{1}-1}-\left(\frac{3}{c_{1}-1}+\frac{2 \mathrm{e}^{\frac{c_{1}}{2}}-6}{c_{1}-2}\right)\right. \\
& \left.+\sum_{n=1}^{\infty} \frac{\left(c_{1}^{n+1} /\left(c_{1}-2\right)-\left(c_{1} / 2\right)^{n+1} /\left(\left(c_{1} / 2\right)-1\right)\right)}{n(n+1)(n+1) !}\right\}
\end{aligned}
$$

Set $p\left(c_{1}\right)=\frac{c_{1}}{4 e^{c_{1}}} \cdot\left(\frac{3}{c_{1}-1}+\frac{2 e^{c_{1} / 2}-6}{c_{1}-2}\right), q\left(c_{1}\right)=\frac{c_{1}}{4 e^{c_{1}}} \cdot \sum_{n=1}^{\infty} \frac{c_{1}^{n+1} /\left(c_{1}-1\right)-\left(c_{1} / 2\right)^{n+1} /\left(c_{1} / 2-1\right)}{n(n+1)(n+1) !}$, $p\left(c_{1}\right)$ and $q\left(c_{1}\right)$ are monotonically decreasing in $[10,+\infty)$.

Since $c_{1} \geq 10.892$, we have $0<p(c 1)<2.8 \times 10^{-3}, 0<$ $q\left(c_{1}\right)<3.7 \times 10^{-3}$, and $0<\frac{c_{1}}{4 \mathrm{e}^{c_{1}}} \cdot \ln 2<3.5 \times 10^{-5}$, we have

$$
-2.8 \times 10^{-3}<\frac{c_{1}}{4 e^{c_{1}}} \cdot \ln 2-p\left(c_{1}\right)+q\left(c_{1}\right)<3.7 \times 10^{-3}
$$

According to the computation of MATLAB7.0, 0 $<\frac{c_{1}}{4 e^{c_{1}}} \cdot \ln 2-p\left(c_{1}\right)+q\left(c_{1}\right)<1.5 \times 10^{-3}$, which is small enough to be neglected. Therefore, we finally simplify Eq. (17) as

$$
E\left(P_{b-a}\right)=\frac{c_{1}}{4 e^{c_{1}}} \cdot \frac{e^{c_{1}}}{c_{1}-1}=\frac{R_{0}^{2}}{4\left(R_{0}^{2}-\delta^{2}\right)}
$$

The probability $P$ that one node within the sensing area of a sensor node has been covered by one of the neighbor sensors is

$$
P=E\left(P_{b-a}\right)
$$

Substitute Eq. (19) into Eq. (18) and the proof of Lemma1 is completed.

Theorem 2 A sensor node expectation of the node redundancy degree with $n$ working neighbor sensor node is 


$$
E\left(\xi_{n}\right)=1-\left(\frac{3 R_{0}^{2}-4 \delta^{2}}{4\left(R_{0}^{2}-\delta^{2}\right)}\right)^{n}
$$

Proof Assuming that the probability that one node within the sensing range of a sensor has been covered by one of the neighbor sensors node is $P$, the probability that this node is not covered is $\vee P$ where $\vee P=1 \times P$. If a sensor has $n$ neighbor sensors, the probability that one node within the sensing area of this sensor node has been not covered by an arbitrary one of the neighbor sensors is $\vee P_{n}$. Since the locations of all the nodes are mutually independent:

$$
\vee P_{n}=(\vee P)^{n}
$$

The probability that one node within the sensing range of this sensor node has been covered by at least one of the neighbor sensing nodes is $P_{n}$ :

$$
P_{n}=1-\vee P_{n}=1-(1-P)^{n}
$$

Suppose a sensor has $n$ working neighbor sensors, the overlapped sensing area between this sensor and its neighbors is $S^{\prime}$. Then, the expectation of $S^{\prime}$ is

$$
E\left(\operatorname{area}\left(S^{\prime}\right)\right)=P_{n} \cdot \operatorname{area}(S)
$$

Here, area $(S)$ is the sensing area of this sensor. So, the expectation for the redundancy degree of a node with $n$ neighbor workers is

$$
E\left(\xi_{n}\right)=\frac{E\left(\operatorname{area}\left(S^{\prime}\right)\right)}{\operatorname{area}(S)}=1-(1-P)^{n}
$$

Substituting the conclusion in Theorem 1 into Eq. (23), we have $E\left(\xi_{n}\right)=1-\left(\frac{3 R_{0}^{2}-4 \delta^{2}}{4\left(R_{0}^{2}-\delta^{2}\right)}\right)^{n}$.

Therefore, the proof of Theorem 2 is completed.

Table 1 illustrates the relationship between the expectation of redundancy degree, and the number of sensing neighbors when Theorem 2 is employed. Observe that the expectation of redundancy degree $E(\xi)$ is deeply affected by the number of its sensing neighbors while the value of $R_{0} / \delta$ shows a minor impact. When the number of working neighbor sensors is no smaller than 13, the expectation for the node redundancy degree can be higher than 95\%. By applying Theorem 2, the sensor node could quickly calculate the expectation of own redundancy degree. Then, the sensor node decides whether it is a redundant node by comparing the redundancy degree and the expectation of QoC.

Theorem 3 If $k$ nodes are randomly chosen as working nodes in the monitoring area $M$, the expectation of the $\mathrm{QoC}$ of $M$ is
Table 1 The expectation of redundancy nodes and the number of sensing neighbors

\begin{tabular}{llllll}
\hline \multirow{2}{*}{$\begin{array}{l}\text { Sensor } \\
\text { neighbors }\end{array}$} & \multicolumn{5}{l}{ Expectation of redundancy nodes $\left(R_{0}=10\right) / \%$} \\
\cline { 2 - 6 } & $\delta=0$ & $\delta=1$ & $\delta=2$ & $\delta=3$ & $\delta=4$ \\
\hline 6 & 72.36 & 73.41 & 73.98 & 74.33 & 75.08 \\
7 & 72.92 & 74.26 & 75.03 & 75.86 & 76.29 \\
8 & 74.12 & 74.95 & 76.21 & 77.08 & 78.13 \\
9 & 78.89 & 80.06 & 82.56 & 83.91 & 85.49 \\
10 & 85.16 & 87.42 & 89.51 & 90.61 & 92.07 \\
11 & 91.63 & 93.08 & 94.87 & 95.69 & 97.80 \\
12 & 94.06 & 96.50 & 97.28 & 98.57 & 98.82 \\
13 & 95.74 & 97.05 & 98.25 & 98.97 & 99.03 \\
14 & 96.09 & 97.71 & 98.68 & 99.02 & 99.51 \\
15 & 97.15 & 98.28 & 99.36 & 99.57 & 99.86 \\
\hline
\end{tabular}

$$
E\left(\eta_{k}\right)=1-\left(1-\frac{\pi\left(R_{0}^{2}+\delta^{2}\right)-\sqrt{2 \pi} R_{0} \mathrm{e}^{-\frac{R_{0}}{2 \delta^{2}}}}{\operatorname{area}(M)}\right)^{k}
$$

Proof Since all the sensing nodes are randomly deployed within the monitoring area $M$, the probability for a node to be deployed at a certain point in $M$ is 1 /area $(M)$. If a node $q$ in $M$ is covered by a sensor $a$ whose sensing range is $R_{a}$, $a$ must lie within the circle $\Theta q\left(R_{a}\right)$ which has center $q$ and radius $R_{a}$. Therefore, the probability for node $q$ to be covered by sensor $a$ is

$$
P_{a}=\frac{\operatorname{area}\left(\Theta q\left(R_{a}\right)\right)}{\operatorname{area}(M)}=\frac{\pi R_{a}^{2}}{\operatorname{area}(M)}
$$

Since the sensing ranges of all the sensors satisfy the normal distribution $N\left(R_{0}, \delta^{2}\right)$ with $R_{0} \geq 3.3 \delta$, the probability for a node in $M$ to be covered by a working node is:

$$
P=E\left(P_{a}\right)=\int_{0}^{2 R_{0}} \frac{\pi R_{a}^{2}}{\operatorname{area}(M)} \cdot \frac{1}{\sqrt{2 \pi} \delta} e^{-\frac{\left(R_{a}-R_{0}\right)^{2}}{2 \delta^{2}}} \mathrm{~d} R_{a}
$$

Set $x=\left(R_{a}-R_{0}\right) / \delta$, and we have:

$$
\begin{aligned}
& P=\frac{1}{\operatorname{area}(M)} \sqrt{\frac{\pi}{2}}\left(\int_{-\frac{R_{0}}{\delta}}^{\frac{R_{0}}{\delta}} \delta^{2} x^{2} e^{-\frac{x^{2}}{2}} \mathrm{~d} x+\int_{-\frac{R_{0}}{\delta}}^{\frac{R_{0}}{\delta}} 2 \delta x R_{0} e^{-\frac{x^{2}}{2}} \mathrm{~d} x\right. \\
& \left.+\int_{-\frac{R_{0}}{\delta}}^{\frac{R_{0}}{\delta}} R_{0}^{2} e^{-\frac{x^{2}}{2}} \mathrm{~d} x\right)=\frac{1}{\operatorname{area}(M)} \sqrt{\frac{\pi}{2}}\left(-\delta^{2} x e^{-\frac{x^{2}}{2} \mid \begin{array}{l}
R_{0} / \delta \\
-R_{0} / \delta
\end{array}+\sqrt{2 \pi} R_{0}^{2}}\right) \\
& =\frac{\pi\left(R_{0}^{2}+\delta^{2}\right)-\sqrt{2 \pi} R_{0} \delta e^{-R_{0}^{2} / 2 \delta^{2}}}{\operatorname{area}(M)}
\end{aligned}
$$

Since all the nodes in $M$ are independently and randomly deployed, the coverage probability that a sensor 
node has be covered by at least one of the $k$ working nodes in $M$.

$$
\begin{aligned}
P_{k} & =\sum_{i=1}^{k}\left(\begin{array}{c}
k \\
i
\end{array}\right) P^{i}(1-P)^{k-i} \\
& =1-\left(1-\frac{\pi\left(R_{0}^{2}+\delta^{2}\right)-\sqrt{2 \pi} R_{0} \delta e^{-R_{0}^{2} / 2 \delta^{2}}}{\operatorname{area}(M)}\right)^{k}
\end{aligned}
$$

Suppose that $M^{\prime}$ is the area within which all the sensor nodes has been covered by at least one of the $k$ working sensor nodes, the expectation for the size of $M^{\prime}$ is

$$
\begin{aligned}
& E\left(\operatorname{area}\left(M^{\prime}\right)\right)=P_{k} \operatorname{area}(M) \\
& \begin{aligned}
E\left(\eta_{k}\right) & =\frac{E\left(\operatorname{area}\left(M^{\prime}\right)\right)}{\operatorname{area}(M)} \\
& =1-\left(1-\frac{\pi\left(R_{0}^{2}+\delta^{2}\right)-\sqrt{2 \pi} \cdot R_{0} \delta \cdot e^{-R_{0}^{2} /\left(2 \delta^{2}\right)}}{\operatorname{area}(M)}\right)^{k}
\end{aligned}
\end{aligned}
$$

This completes the proof.

\subsection{Process of NECP implementation}

The ENCP employs the distributive scheduling algorithm, i.e., each node compares the desired QoC $\eta_{d}$ with its own expectation of redundancy degree $E(\xi)$. If $E(\xi) \geq \eta_{d}$, we have $1-\left(3 R_{0}^{2}-4 \delta^{2} / 4\left(R_{0}^{2}-\delta^{2}\right)\right)^{n} \geq \eta_{d}$ according to Theorem 2. That is to say, the QoC of the sensing area of this node satisfies the desired QoC, and this node can be shut down to save the network energy consumption. In other words, if the number of sensing neighbors $n$ for a certain node satisfies

$$
n \geq \frac{\ln \left(1-\eta_{d}\right)}{\ln \left(\left(3 R_{0}^{2}-4 \delta^{2}\right) /\left(4\left(R_{0}^{2}-\delta^{2}\right)\right)\right)}
$$

This node is then regarded as a redundant node which can be shut down to save energy.

The initial power is equal for all the nodes. A management node is randomly chosen in an arbitrary alliance. Centered by this management node, the routing protocol is transmitted in a single-hop or multiple-hop method. Assuming that the management node is deployed in advance with unlimited power, it could communicate directly with all the member nodes. However, the member nodes need to perform single-hop or multiple-hop routing to reach the management node. This is an optimization problem which minimizes the cost from the source to the destination. Since the energy consumption models, i.e., the calculation model for the communication energy, the calculation model for the data processing energy and the calculation model for the environment sensing energy, are definite, upon receiving one message, the management node could trace the energy change of all the nodes processing this message according to energy models, the message length and the data quantity. For each round of cycles, the nodes in the alliance are divided into three states according to the protocol, i.e., work, election, and sleep. Sensing nodes only take charge of monitoring the environment and collecting data. Relay nodes are in charge of relaying data while sensing relay nodes possess both of these two functions. When inactive nodes are switched to the sleep state, the management node determines the state of each node according to the node energy, topology, and network task. When the routing is established, the management node broadcasts the node state and routing information to all the nodes. Due to message loss and data processing delay, there may be errors in the energy calculation model for the management node. Therefore, the member nodes are required to transmit the energy update message on the present energy to the management nodes. Then, the optimal routing is calculated and broadcasted to the members in the alliance by the management node.

The ENCP divides the network running time into several rounds, each of which consists of the coverage control period and the steady state period. During the coverage control period, the functioning modules of all the redundant nodes are shut down to save energy. During the steady state period, the remaining working nodes perform the regular monitoring and communication. Each node has six running states, i.e., election state, Start_working state, Start_sleeping state, back_off state, election, work, and sleep. The transition diagram between the six states is depicted in Fig. 4. When the node density is high, almost all the nodes satisfy Eq. (31) and they will try to switch to the sleep state. However, when the neighbor nodes switch to the sleep state simultaneously, coverage dead zones will exist, and thus, the QoC is undermined. To avoid this case, the ENCP employs the back_off scheme and the scheme which directly reduces the node density. At the election state, the density of potential working nodes is firstly reduced, i.e., $k$ nodes are directly elected as candidate working nodes while the others are switched to the sleep state. Then, the redundant node scheduling is performed among the $k$ candidate nodes. Each candidate elects itself to the start_work state with probability $P$ while the other failed candidates switch to the start_sleep state.

\subsection{Set value of $k$}

At the beginning, since all the nodes have the same initial energy and the management node is chosen 


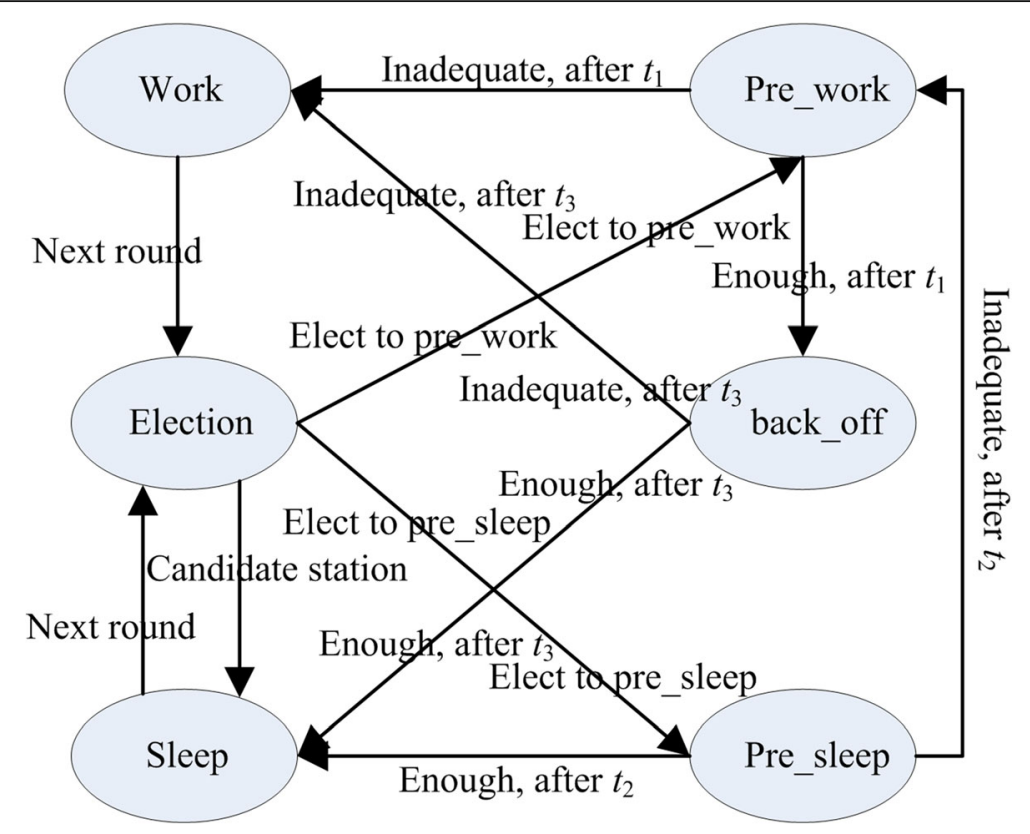

Fig. 4 Finite state machine of ENCP

randomly, the energy are consumed by all the nodes to some extent after one or several working rounds. According to the proposed algorithm, the node with more remained energy is chosen as the management node for the next round. Meanwhile, the rank of all the nodes is updated in the link. During the establishment of the alliance, each node randomly chooses a real number between 0 and 1 . If this number is smaller than a certain threshold, this node is chosen as the management node. Then, the identification of this management node is broadcasted to all the nodes. Each node determines its own alliance according to the amplitude of the receiving signal and broadcasts its membership to all of the alliance members. During the data transmission period, all the member nodes transmit data to the management nodes in Time Division Multiple Access (TDMA) slots while the management nodes integrate the receiving data and broadcast them to the base station. After several working rounds, the network is restarted. Then, the next round of election for the management nodes is performed and the new alliances are established.

At the beginning of each round, in order to reduce the density of working sensor node, $k$ nodes are firstly chosen as candidates. Therefore, $k$ nodes should not be too dense or too sparse. $k$ value should be at least larger than the minimal number of working sensor nodes which could guarantee the expectation of QoC.

Since $\sqrt{2 \pi} \cdot R_{0} \delta \cdot \mathrm{e}^{-R_{0}^{2} /\left(2 \delta^{2}\right)} / \pi\left(R_{0}^{2}+\delta^{2}\right) \leq 0.1 \times 10^{-3}$, the term $\sqrt{2 \pi} \cdot R_{0} \delta \cdot \mathrm{e}^{-R_{0}^{2} /\left(2 \delta^{2}\right)}$ has been neglected in practice in Theorem 3 to simplify the calculation, i.e.,

$$
E\left(\eta_{k}\right)=1-\left(1-\frac{\pi\left(R_{0}^{2}+\delta^{2}\right)}{\operatorname{area}(M)}\right)^{k}
$$

According to Eq. (32), we can derive the expectation of the minimal working sensor nodes to guarantee the expectation of QoC,

$$
E(k)=\frac{\ln \left(1-\eta_{d}\right)}{\ln \left(1-\pi \cdot\left(R_{0}^{2}+\delta^{2}\right) / \operatorname{area}(M)\right)}
$$

In ENCP, we choose $K=\lceil 2 E(k)\rceil$ so that globally, it can be guaranteed that there are enough nodes to satisfy the desired QoC. Then, by the local node scheduling of the ENCP, redundant nodes are switched to the sleep node to achieve the uniform coverage within the monitoring range. To balance the node energy consumption in the network, the $K$ candidate nodes are chosen in the same way the cluster heads are chosen in [24].

\section{ENCP evaluation systems}

In order to verify the validity and performance for the ENCP, we perform simulations based on the OPNET Modeler platform. The monitoring area for the simulation is $200 \times 200 \mathrm{~m}^{2}$ and the sensing ranges of the nodes satisfy the normal distribution $N\left(10, \delta^{2}\right)$, where $10 \geq 3.3 \delta$. The node energy consumption model is the same as the physical model in [25], i.e., the energy consumption ratio for the transmitting state. The transmission rate for the node is $56 \mathrm{~kb} / \mathrm{s}$. The time for each round is $200 \mathrm{~s}$ while the durations for $T_{1}, T_{2}$, and $T_{3}$ are $5 \mathrm{~s}$. The probability $P$ for each candidate node to elect 


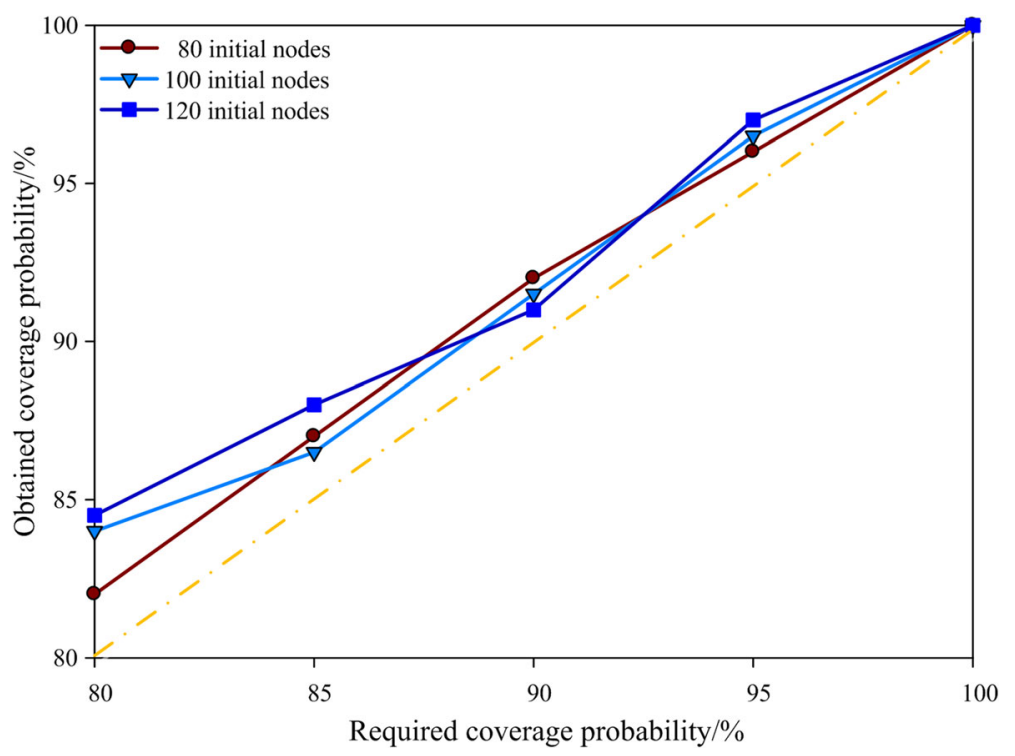

Fig. 5 Relationship between required QoC and obtained QoC when $\delta=3$

itself into the start_work state is $10 \%$. The initial energy for each node is sufficient to last 190-210 rounds of consistent receiving state.

An ideal coverage control algorithm should utilize the minimal number of working nodes to satisfy the desired QoC, which could save the network energy consumption as much as possible. The relationship between the required $\mathrm{QoC}$ and the obtained $\mathrm{QoC}$ is depicted in Fig. 5 for different number of deployed nodes when $\delta=3$. It can be observed that regardless of the number of deployed nodes, the ENCP could always guarantee the required QoC. Since the number of sensing neighbors is definitely an integer, the obtained $\mathrm{QoC}$ is a little higher than the required QoC. However, the difference between the required and the obtained QoC quickly reduces to 0 as the required QoC increases. Furthermore, it is also impractical to obtain the exact required $\mathrm{QoC}$ when the location information is not available.

The relationship between the number of working nodes and the number of deployed nodes is depicted in Fig. 6 when $\delta=3$. It can be observed from Fig. 6 that the number of working nodes is only related with the desired QoC, instead of the number of deployed nodes. Furthermore, the number of working nodes is almost equal to the

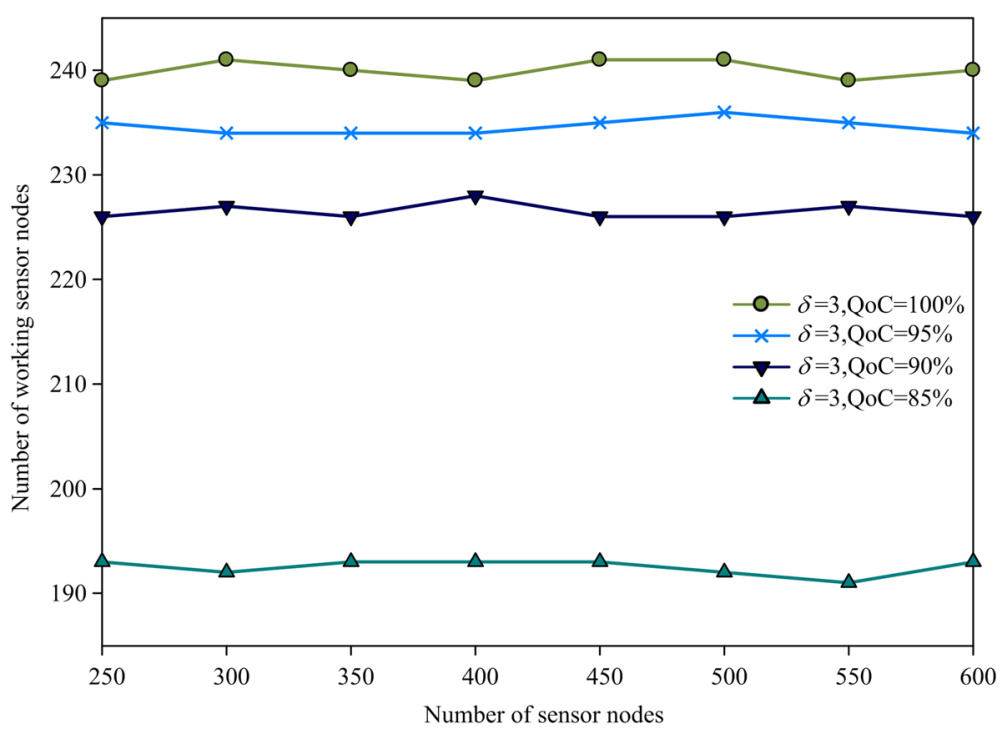

Fig. 6 Relationship between the numbers of working nodes and deployed nodes when $\delta=3$ 


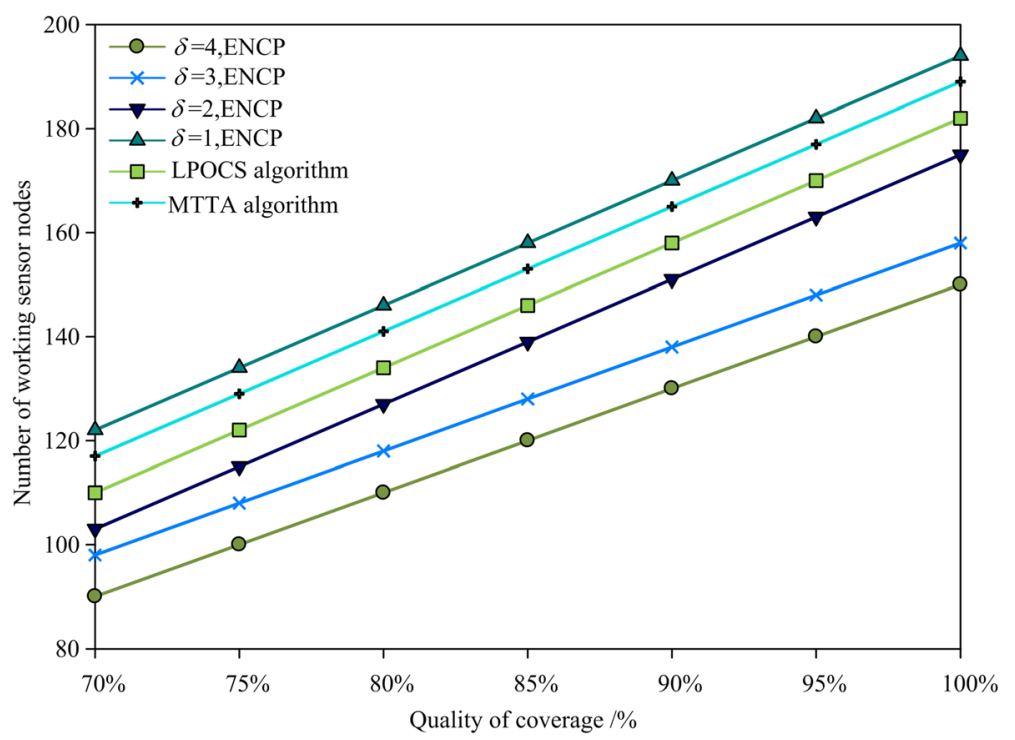

Fig. 7 Required QoC and obtained QoC with different $\delta$

result calculated according to Eq. (33). This observation verifies the excellent scalability of the ENCP. Although the LDAS [28] shows similar scalability, much more working nodes are needed for the LDAS to guarantee the same QoC. The reason behind this will be explained in Section 4.1. We can also observe from Fig. 6 that more working nodes are required for a higher required QoC.

The relationship between the required $\mathrm{QoC}$ and the obtained QoC for different values of $\delta$ is depicted in Fig. 7. It can be observe from Fig. 7 that the obtained QoC matches well with the required QoC regardless of the value of $\delta$. This observation proves that this protocol can be well applied to any MSNs where the sensing ranges satisfy the normal distribution $N\left(R_{0}, \delta^{2}\right)$ with $R_{0} \geq 3.3 \delta$.

To further verify the enhancement of the equalization for the network power, we compare the ENCP with the algorithms in $[18,19]$. The simulations are performed based on three different sensing fields, and the coverage probability is fixed to $99.9 \%$. The simulation results for different parameters are depicted in Figs. 8, 9, 10, and 11.

Figures $8,9,10$, and 11 depict the relationship between the network lifetime and the target nodes within

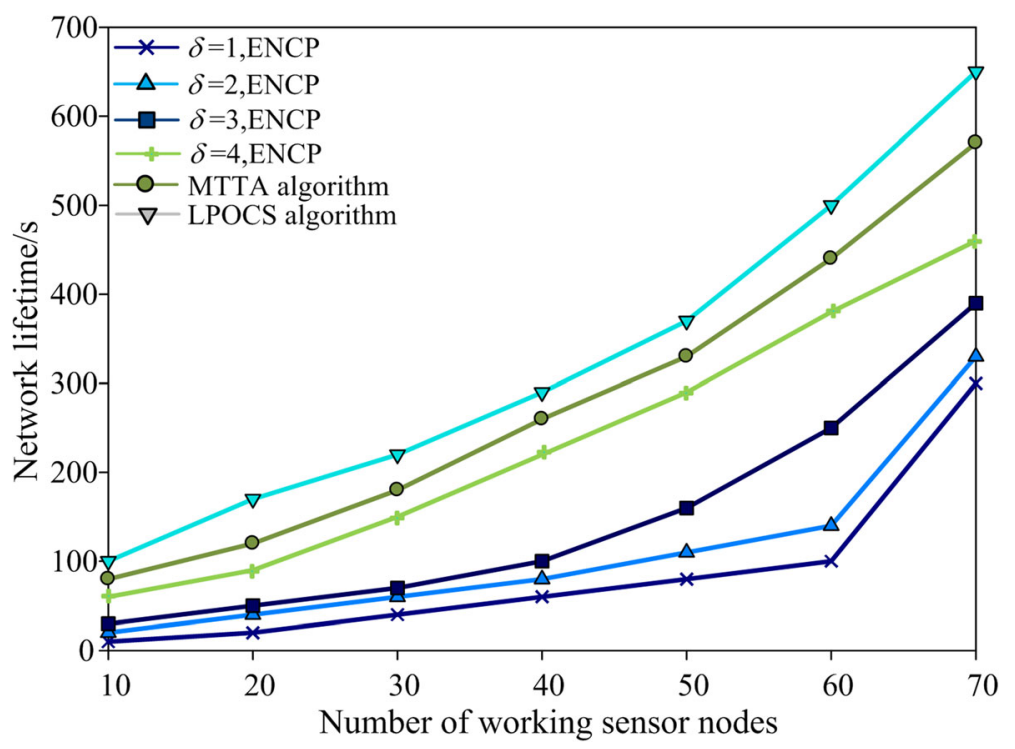

Fig. $8100 \times 100 \mathrm{~m}^{2}$, network lifetime vs the number of working sensor nodes 


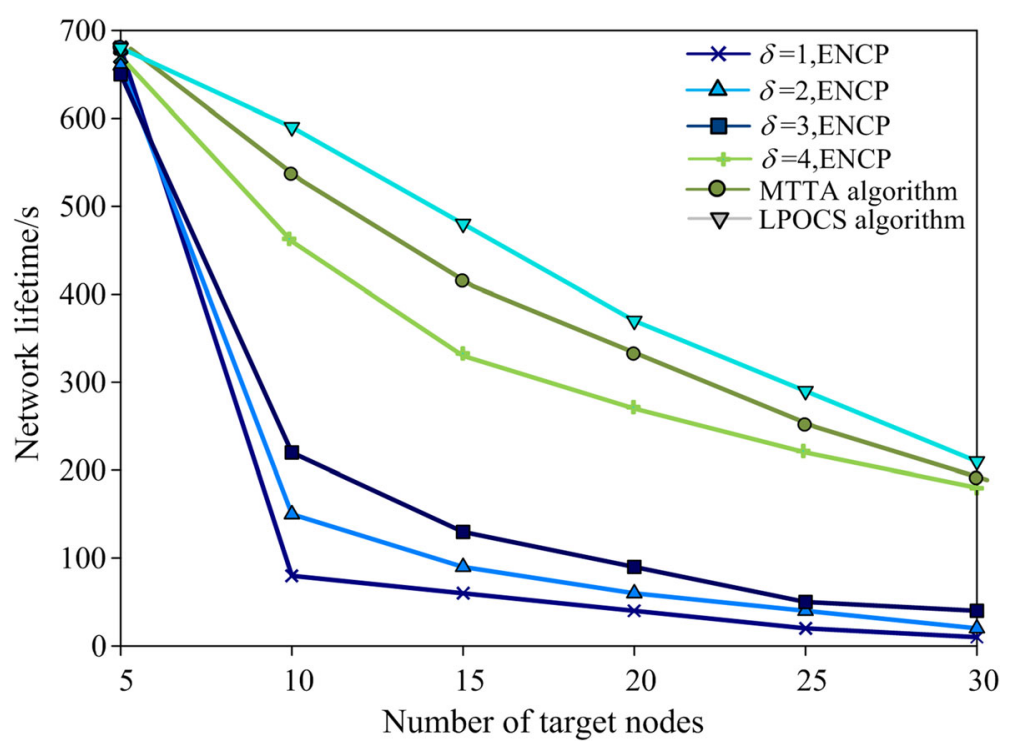

Fig. $9100 \times 100 \mathrm{~m}^{2}$, network lifetime vs the number of target nodes

different sensing fields. The algorithms we focus on are the Multiple Target Tracking Algorithm (MTTA) [18] and linear programming optimization coverage scheme (LPOCS) [19]. It can be observed from Fig. 8 that at the initial stages of the program, the network lifetime of the three algorithms increases with the number of nodes. However, due to the limit of the value range of this algorithm and the inactive state of the redundant nodes, the network lifetime of the ENCP is lower than the other algorithms when the equalization is finally achieved for the network energy. During the coverage process for the target node, less network energy is required for the ENCP, which can be explained as above. In Fig. 10, parts of the redundant nodes are transitioned to the state of work due to the increase of the area of the sensing fields. As a result, the network lifetime is prolonged. When $\delta=3$, the network lifetime of the ENCP is longer than that of the LPOCS algorithm. When $\delta=4$, the network

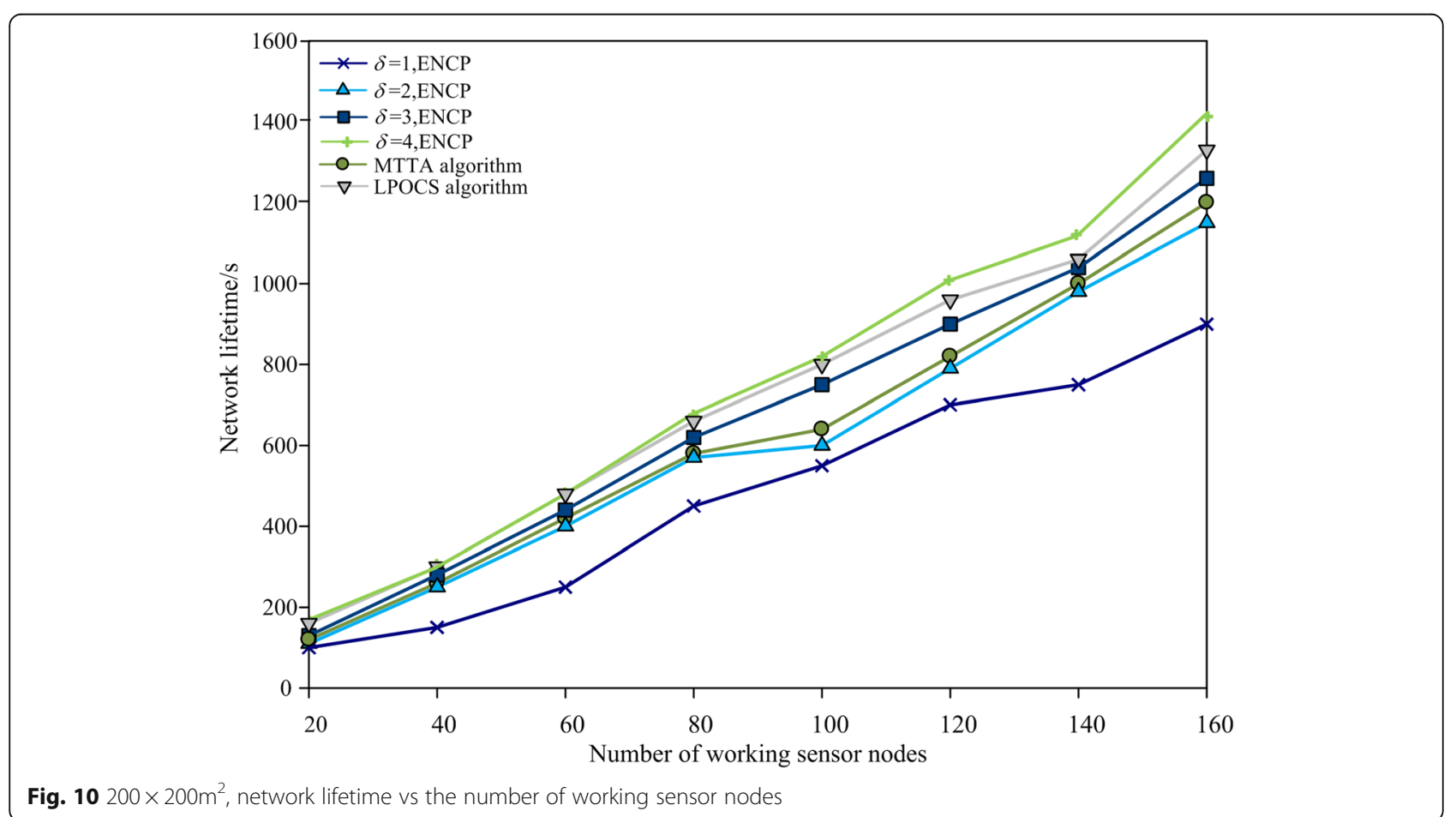

Fig. $10200 \times 200 \mathrm{~m}^{2}$, network lifetime vs the number of working sensor nodes 


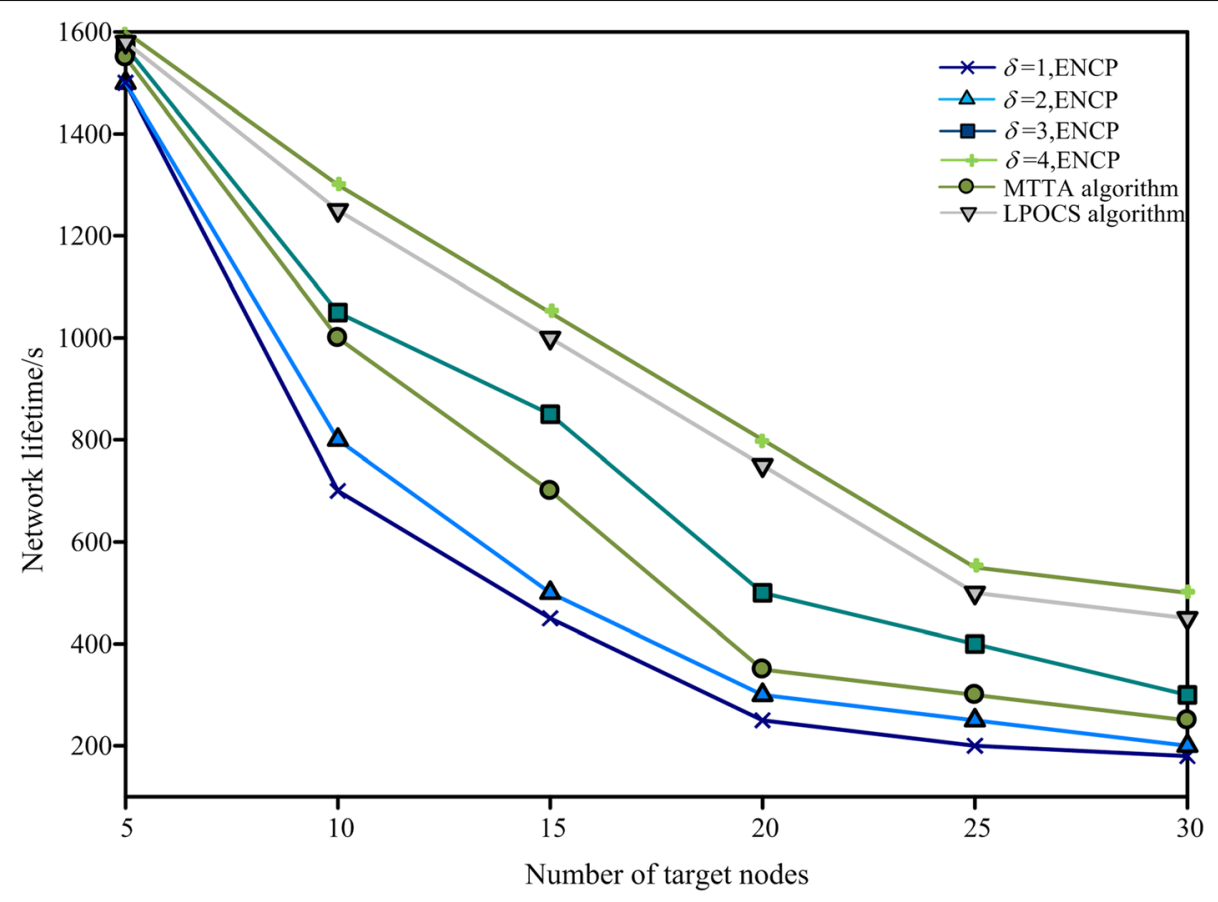

Fig. $11200 \times 200 \mathrm{~m}^{2}$, network lifetime vs the number of target nodes

lifetime of the ENCP is longer than those for both of the other two algorithms. The network lifetime during the coverage for the target node is depicted in Fig. 11. It can be observed that the network lifetime of three algorithms decreases with the increase of target nodes. Finally, the energy tends to be equalized. However, the ENCP exhibits a lower decrease speed during the decline process. This is due to the fact that when a part of the sensing field is densely deployed with the sensor nodes, i.e., the coverage expectation is higher for this region, some redundant nodes are awakened via the scheduling mechanism of the sensor nodes. These awakened nodes are transitioned to the state of work to enhance the coverage intensity and further prolong the network lifetime.

\section{Conclusions}

Focusing on the sensing characteristics of randomly deployed MSNs, we analyzed the coverage redundancy problem for the MSNs where the sensing ranges satisfy the normal distribution. We also presented the calculation model for the node redundancy degree for which no location information is needed and the calculation model for the minimal number of working nodes to guarantee the network QoC. According to the analytical result, we proposed the ENCP which shut down all the redundant nodes satisfying the redundancy condition. Based on the ENCP, it enables the collaborative scheduling of distributed sensor nodes and balances the energy consumptions of each sensor nodes. The purpose of energy conservation of the networks is achieved since the ENCP maintains the least number of sensor nodes, as working nodes to provide the desired QoC. Simulation results show that the ENCP could not only accurately guarantee the desired $\mathrm{QoC}$, but also efficiently reduce the network energy consumption to prolong the effective network lifetime.

\section{Acknowledgements}

This work was supported by the National Natural Science Foundation of China under grant no. 61628210, Henan Province Education Department Cultivation Young Key Teachers in University of under grant no. 2016GGJS158, Henan Province Education Department Natural Science Foundation under grant no. 17A520044, Luoyang Institute of Science and Technology High-level Research Start Foundation under grant no. 2017BZ07, Natural Science and Technology Research of Henan Province Department of Science Foundation under grant no. 162102210113, Guangdong Natural Science Foundation of China under grant no. 2016A030313540, Guangzhou

Education Bureau Science Foundation under grant no. 1201430560, Science and Technology Planning Project of Guangzhou under grant no.

201707010284, and Shaanxi Education Bureau Science Foundation under grant no. 2016SF-428. Finally, great thank to the anonymous reviewers for their valuable suggestions to improve the quality of the paper.

\section{Funding}

This work was sponsored by the National Natural Science Foundation of China under grant no. 61628210, Henan Province Education Department Cultivation Young Key Teachers in University of under grant no. 2016GGJS158, Henan Province Education Department Natural Science Foundation under grant no. 17A520044, Luoyang Institute of Science and Technology High-level Research Start Foundation under grant no. 2017BZ07, Natural Science and Technology Research of Henan Province Department of Science Foundation under grant no. 162102210113, Guangdong Natural Science Foundation of China under grant no. 2016A030313540, Guangzhou Education Bureau Science Foundation under grant no. 1201430560, Science and Technology Planning Project of Guangzhou under grant no. 
201707010284, and Shaanxi Education Bureau Science Foundation under grant no. 2016SF-428.

\section{Authors' contributions}

ZYS and XFX contributed to the conception and algorithm design of the study. ZYS and GZZ contributed to the acquisition of the simulation. All authors contributed to the analysis of the simulation data and approved the final manuscript.

\section{Authors' information}

Zeyu Sun received a M.S. and Ph.D degree from Lanzhou University and Xi'an Jiaotong University, in 2010 and 2017. He is an assistant professor in the School of Computer and Information Engineering, Luoyang Institute of Science and Technology, Luoyang, Henan, China. He is a member of China Computer Federation. His research interests lie in wireless sensor networks, parallel computing, mobile computing, and Internet of things (e-mail: lylgszy@163.com). Guozeng Zhao received a M.S. degree from Taiyuan University of Technology in 2010. He is a lecturer in School of Computer and Information Engineering, Luoyang Institute of Science and Technology, Luoyang, China. His research interests include wireless sensor networks and cloud computing (e-mail:ly_zgz@163.com).

Xiaofei Xing received BS degree in computer science and technology from Henan University of Science and Technology in 2003, and an MS and Ph.D. degrees in Central South University, China, in 2008 and 2012, respectively. He has been a Research Fellow at University of Tsukuba, Japan, and he had been also a post-doctorate researcher in Applied Mathematics. He is an assistant professor in the School of Computer Science and Educational Software, Guangzhou University, Guangzhou, China. His research interests include wireless sensor networks, mobile computing, and network performance analysis (e-mail:xxfcsu@163.com).

\section{Competing interests}

The authors declare that they have no competing interests.

\section{Publisher's Note}

Springer Nature remains neutral with regard to jurisdictional claims in published maps and institutional affiliations.

\section{Author details}

'School of Computer Science and Engineering, Luoyang Institute of Science and Technology, Luoyang, China. ${ }^{2}$ Department of Computer Science and Technology, Xi'an Jiaotong University, Xi'an, China. ${ }^{3}$ School of Computer Science and Educational Software, Guangzhou University, Guangzhou, China.

\section{Received: 17 October 2017 Accepted: 2 January 2018}

\section{Published online: 15 January 2018}

\section{References}

1. N Mittal, U Singh, BS Sohi, A stable energy efficient clustering protocol of wireless sensor networks. Wirel. Netw 23(6), 1809-1821 (2017). https://doi. org/10.1007/s11276-016-1255-6

2. V Kumar, SB Dhok, R Tripathi, S Tiwari, Cluster size optimization with Tunable Elfes sensing model for single and multi-hop wireless sensor networks. Int. J. Electron. 104(2), 312-327 (2017). https://doi.org/10.1080/ 00207217.2016. 1216177

3. Z Zhao, J Willson, L Zaixin, W Weili, Z Xuding, D Dingzhu, Approximating maximum lifetime $k$-coverage through minimizing weighted $k$-cover in homogeneous wireless sensor networks. IEEE/ACM Trans. Networking 24(6), 3620-3633 (2016). https://doi.org/10.1109/TNET.2016.2531688

4. J Habibi, H Mahboubi, AG Aghdam, Distributed coverage control of mobile sensor networks subject to measurement error. IEEE Trans. Autom. 61(11), 3330-3343 (2016). https://doi.org/10.1109/TAC.2016.2521370

5. L Saewoom, K Kiseon, Key renewal scheme with sensor authentication under clustered wireless sensor networks. Electron. Lett. 51(4), 368-369 (2015). https://doi.org/10.1049/el.2014.3327

6. AK Idrees, K Deschinkel, M Salomon, C Raphael, Perimeter-based coverage optimization to improve lifetime in wireless sensor networks. Eng. Optim. 48(11), 1951-1972 (2016). https://doi.org/10.1080/0305215X.2016.1145015

7. N Xiong, V Athanasios, L Yang, L Song, Y Pan, K Rajgopal, Y Li, Comparative analysis of quality of service and memory usage for adaptive failure detectors in healthcare systems. IEEE J. Sel. Areas Commun. 27(4), 495-509 (2009). https://doi.org/10.1109/SAC.2009.090512

8. F Senel, Coverage-aware connectivity-constrained unattended sensor deployment in underwater acoustic sensor networks. Wirel. Commun. Mob. Comput. 16(14), 2052-2064 (2016). https://doi.org/10.1002/WCM.2667

9. K Huang, Z Qi, C Zhou, N Xiong, An efficient intrusion detection approach for visual sensor networks based on traffic pattern learning. IEEE Trans. Syst. Man Cybernetics: Syst. 47(10), 2704-2713 (2017) Doi:10.1109 /TSMC.2017.2698457

10. G Mario, B Holger, S Slawomir, Nomographi functions: efficient computation in clustered Gaussian sensor networks. IEEE Trans. Wirel. Commun. 14(4), 2093-2105 (2015). https://doi.org/10.1109/TWC.2014.2380317

11. M Rout, R Roy, Self-deployment of randomly scattered mobile sensors to achieve barrier coverage. IEEE Sensor J. 16(18), 6819-6820 (2016). https:// doi.org/10.1109/JSEN.2016.2590572

12. M Ozger, E Fadel, OB Akan, Event-to-sink spectrum-aware clustering in mobile cognitive radio sensor networks. IEEE Trans. Mobile Comput. 15(9), 2221-2233 (2016). https://doi.org/10.1109/YMC.2015.2493526

13. D Saha, N Das, Self-organized area coverage in wireless sensor networks by limited node mobility. Innov. Syst. Softw. Eng. 12(3), 227-238 (2016). https:// doi.org/10.1007/s11334-016-0277-7

14. Z Sun, Y Zhang, Y Nie, W Wei, J Lloret, H Song, CASMOC: a novel complex alliance strategy with multi-objective optimization of coverage in wireless sensor networks. Wirel. Netw 23(4), 1201-1222 (2017). https://doi.org/10. 1007/s 11276-016-1213-3

15. $\mathrm{H}-\mathrm{H}$ Cho, TK Shih, $\mathrm{H}-\mathrm{C}$ Chao, A robust coverage scheme for UWSNs using the spline function. IEEE Sensor J. 16(11), 3995-4002 (2016). https://doi.org/ 10.1109/JSEN.2015.2429914

16. N Dong, X Ren, W Wang, M Liu, Node scheduling scheme using spatial resolution of wireless sensor networks. J. Comput. Inf. Syst. 11(10), $3701-$ 3708 (2015). https://doi.org/10.12733/jcis14262

17. X Xing, G Wang, j Li, Polytype target coverage scheme for heterogeneous wireless sensor networks using linear programming. Wirel. Commun. Mob. Comput. 14(14), 1397-1408 (2014). https://doi.org/10.1002/wcm.2269

18. T Wang, Z Peng, J Liang, W Sheng, MD Zakirul Alam Bhuiyan, Y Cai, J Cao, Follow targets for mobile tracking in wireless sensor networks. ACM Trans. Sens. Networks 12(4), 31.1-31.24 (2016). https://doi.org/10.1145/2968450

19. Z Sun, Y Shu, X Xing, W Wei, H Song, W Li, LPOCS: a novel linear programming optimization coverage scheme in wireless sensor networks. Ad Hoc \& Sens. Wireless Networks 33(1-4), 173-197 (2016)

20. Z Bo, E Tong, J Hao, W Niu, G Li, Energy efficient sleep schedule with service coverage guarantee in wireless sensor networks. J. Netw. Syst. Manag. 24(4), 834-858 (2016). https://doi.org/10.1007/s10922-015-9361-9

21. ZSW Wu, H Wang, $H$ Chen, Xiaofei, A novel coverage algorithm based on event-probability-driven mechanism in wireless sensor network. EURASIP J. Wireless Commun. Networking 58, 1-16 (2014) Doi: 10. 1186/1687-14992014-58

22. Z Sun, R Tao, L Li, X Xing, A new energy-efficient multi-target coverage control protocol using event-driven-mechanism in wireless sensor networks. Internet J. Online Eng. 13(2), 53-67 (2017). https://doi.org/10.3991/ijoe.v13 i02.6465

23. G Xing, M Li, T Wang, W Jia, J Huang, Efficient rendezvous algorithms for mobility-enabled wireless sensor networks. IEEE Trans. Mob. Comput. 11(1), 47-60 (2012). https://doi.org/10.1109/TMC.2011.66

24. K Lin, X Tianlang, J Song, Y Qian, Y Sun, Node scheduling for all-directional intrusion detection in SDR-based 3D WSNs. IEEE Sens. J. 16(20), 7332-7341 (2016). https://doi.org/10.1109/JSEN.2016.2558043

25. JP Mohanty, C Mandal, C Reade, A Das, Construction of minimum connected dominating set in wireless sensor networks suing pseudo dominating set. Ad Hoc Netwroks 42(2), 61-73 (2016). https://doi.org/10. 1016/j.adhoc.2016.02.003

26. HS Aghdasi, M Abbaspour, Energy efficient area coverage by evolutionary camera node scheduling algorithm in visual sensor networks. Soft. Comput. 20(3), 1191-1202 (2016). https://doi.org/10.1007/s00500-014-1582-4

27. S Jamali, M Hatami, Coverage aware scheduling in wireless sensor networks: an optimal placement approach. Wirel. Pers. Commun. 85(3), 1689-1699 (2015). https://doi.org/10.1007/s11277-015-2862-8

28. D Zeng, P Li, S Guo, T Miyazaki, H Jiankun, Y Xiang, Energy minimization in multi-task software-defined sensor networks. IEEE Trans. Comput. 64(11) 3128-3139 (2015). https://doi.org/10.1109/TC.2015.2389802

29. W Kui, Y Gao, F Li, Y Xiao, Lightweight deployment-aware scheduling for wireless sensor networks. Mobile Networks Appl. 10(6), 837-852 (2005). https://doi.org/10.1007/s11036-005-4442-8 
30. C-p Chen, S Mukhopadhyay, C-I Chuang, T-S Lin, M-S Liao, Y-C Wang, J-A Jiang, A hybrid memetic framework for coverage optimization in wireless sensor networks. IEEE Trans. Cybernetics 45(10), 2309-2322 (2015). https:// doi.org/10.1109/TCYB.2014.2371139

31. C-F Cheng, C-W Huang, An energy-balanced and timely self-relocation algorithm for grid-based mobile WSNs. IEEE Sensors J. 15(8), 4184-4193 (2015). https://doi.org/10.1109/JSEN.2015.2413367

32. Q Gao, W Ma, W Luo, A combinatorial key predistribution scheme for twolayer hierarchical wireless sensor networks. Wirel. Pers. Commun. 96(2), 2179-2204 (2017). https://doi.org/10.1007/s11277-017-4292-2

33. G Mali, S Misra, TRAST: trust-based distributed topology management for wireless multimedia sensor networks. IEEE Trans. Comput. 65(6), 1978-1991 (2016). https://doi.org/10.1109/TC.2015.2456026

34. H Wang, F Meng, Z Li, Energy efficient coverage conserving protocol for wireless sensor networks. J. Software (China) 21(12), 3124-3137 (2010). https://doi.org/10.3724/SP.J.1001.2010.03693

35. X Jing, H Hanwen, H Yang, A Man Ho, S Li, N Xiong, I Muhammad, V Vasilakos Athanasios, A quantitative risk assessment model involving frequency and threat degree under line-of-business services for infrastructure of emerging sensor networks. Sensor 17(3), 642 (2017). https://doi.org/10.3390/s17030642

36. S Aamir, L Malrey, L Changhoon, N Xiong, K Suntae, L Youngkeun, K Kangmin, W Seonmi, J Gisung, The protocol design and new approach for SCADA security enhancement during sensors broadcasting system. Multimedia Tools Appl. 75(22), 14641-14668 (2016). https://doi.org/10.1007/ s11042-015-3050-2

37. Y Yoon, Y-H Kim, An efficient genetic algorithm for maximum coverage deployment in wireless sensor networks. IEEE Trans. cybernetics 43(5), 1473-1483 (2013). https://doi.org/10.1109/TCYB.2013.2250955

38. J Zhang, X Li, S Zhou, X Ye, A novel sleep scheduling scheme in green wireless sensor networks. J. Supercomuting 71(3), 1067-1094 (2015). https:// doi.org/10.1007/s11227-014-1354-z

39. S Wang, $\mathrm{H}$ Yi, L Wu, F Zhou, NN Xiong, Mining probabilistic representative gathering patterns for mobile sensor data. J. Internet Technol. 18(2), 321332 (2017). https://doi.org/10.6138/JIT.2017.18.2.20161125

40. J-W Lee, B-S Choi, J-j Lee, Energy-efficient coverage of wireless sensor networks using ant colony optimization with three types of pheromones. IEEE Trans. Ind. informatics 7(3), 419-427 (2011). https://doi.org/10.1109/TI. 2011.2158836

41. H Mohamadi, AS Ismail, S Salleh, Solving target coverage problem using cover sets in wireless sensor networks based on learning automata. Wirel. Pers. Commun. 75(1), 447-463 (2014). https://doi.org/10.1007/s11277-013$1371-X$

42. $\quad H$ Cheng, S Zhihuang, N Xiong, X Yang, Energy-efficient node scheduling algorithm for wireless sensor networks using Markov random field model. Inf. Sci. 329(2), 461-477 (2016). https://doi.org/10.1016/j.ins.2015.09.039

43. PChen, W Hu, Sleep-wake up scheduling with probabilistic coverage model in sensor networks. International Journal of Parallel, Emergent and Distributed Syst. 29(1), 1-16 (2014). https://doi.org/10.1080/17445760.2013.766733

44. B Bae, J Park, S Lee, A free market economy model for resource management in wireless sensor networks. Wireless Sens. Networks 7(6), 76-82 (2015). https:// doi.org/10.4236/wsn.2015.76007

45. L Zaixin, WW Li, M Pan, Maximum lifetime scheduling for target coverage and data collection in wireless sensor networks. IEEE Trans. Vehicular Technol. 64(2), 714-727 (2015). https://doi.org/10.1109/TVT.2014.2322356

46. N Xiong, RW Liu, M Liang, W Di, Z Liu, W Huisi, Effective alternating direction optimization methods for sparsity-constrained blind image deblurring. Sensors 17(1), 174 (2017). https://doi.org/10.3390/s17010174

\section{Submit your manuscript to a SpringerOpen ${ }^{\circ}$ journal and benefit from:}

- Convenient online submission

- Rigorous peer review

- Open access: articles freely available online

- High visibility within the field

- Retaining the copyright to your article

Submit your next manuscript at $\gg$ springeropen.com 